\title{
Simultaneous Determination of Glycyrrhizin and 15 Flavonoids in Licorice and Blood by High Performance Liquid Chromatography with Ultraviolet Detector
}

\author{
Yongqian Zhang, ${ }^{1}$ Jin Cao, ${ }^{2}$ Yingping Wang, ${ }^{3}$ and Shengyuan Xiao ${ }^{1,3}$ \\ ${ }^{1}$ School of Life Science, Beijing Institute of Technology, Beijing 100081, China \\ ${ }^{2}$ Institute for Food and Cosmetics Control, National Institutes for Food and Drug Control, Beijing 100050, China \\ ${ }^{3}$ Institute of Special Wild Economic Animals and Plants, Chinese Academic of Agricultural Science, Jilin, Jilin City 132109, China \\ Correspondence should be addressed to Shengyuan Xiao; shyxiao@gmail.com
}

Received 8 May 2013; Accepted 11 June 2013

Academic Editors: P. Campíns-Falcó and A. Taga

Copyright (c) 2013 Yongqian Zhang et al. This is an open access article distributed under the Creative Commons Attribution License, which permits unrestricted use, distribution, and reproduction in any medium, provided the original work is properly cited.

\begin{abstract}
Licorice is the most frequently used herb in traditional Chinese medicine (TCM) with versatile functions. It is also a popular natural dietary supplement. While the dosages is very important for there are some side effects caused by licorice. The composition of licorice, its products should be well determined thereof. A simple method for simultaneous determining sixteen compounds in vary high dynamic range of content has been established. This method based on the detection at the characteristic ultraviolet spectra of different types of compounds in licorice. Glycyrrhizin and fifteen flavonoids were well measured. All of these compounds can be precisely quantified at their characteristic wavelengths. This method has been successfully applied to the analyses of different licorices, Sini Tang decoction, and rat plasma after oral administration of Sini Tang decoction. These compounds were found to be over 3000 times in content (from $0.01 \mu \mathrm{g} / \mathrm{g}$ to $34.5 \mu \mathrm{g} / \mathrm{g}$ ) in some samples.
\end{abstract}

\section{Introduction}

It is well known that herbal prescription is becoming increasingly popular as supplementary and alternative medicine in recent years. The herbal medicines are believed to be multitarget therapies in modern medicine [1-4]. Herbal drugs usually consist of a large number of ingredients [5]. These ingredients might interact with each other during the process of decoction, absorption, metabolism, or at physiological processes [6-9]. Multiconstituent detection is very important to uncover these interactions during the process of the herb application. It is also important to summarize the common behavior of some constituents with similar structure during some bioprocesses, which is a crucial method to unveil the bioactive mechanism of a herbal drug [10]. However, there are two kinds of obstacles that should be succeeded for multiple compounds detection. The first is the large number of compounds in the herb. The second is the high dynamic range of their contents. High resolution and peak capacity are needed to resolve the overlaid signals of these compounds.
In order to estimate the different concentrations of them, detector with very long-range linearity and good selectivity of the detector response should be employed.

Licorice is an important herb in oriental medicine. It is widely used to treat gastric ulcer, hepatitis $\mathrm{C}$ and pulmonary and skin diseases [11-15]. Licorice is also a popular natural dietary supplement [16]. According to a statistic investigation [17] on Chinese herbal prescription, licorice is the most frequently used herb in traditional Chinese medicine (TCM). It has been proved that flavonoids and triterpenoids are the main bioactive ingredients in licorice, whereas licorice was proved to have side effects, that is, hypertension [18-22], salt and water retention [23], and low potassium levels [24, 25] if it is overdosed. The major side effect of licorice is due to the cortisol-like structure of triterpenoids $[7,26]$. The phenolic compounds in licorice were also proved to play a dual role [27]. Isoliquiritigenin has also been found to have dual effect in regulating gastrointestinal motility. It showed spasmogenic and spasmolytic effects in different dosages [27]. The contents of these compounds in licorice and its productions as well 
their dosages, should be well controlled thereof. On the other hand, phenolic compounds interfere with the metabolism of triterpenoids. Phenolic compounds in licorice extract can decrease the bioavailability of glycyrrhizin $(G)[7,8]$ and increase the excretion of $\mathrm{G}$ into bile in a rat [7]. Phenolic compounds in licorice can also decrease the hydrolyzation of $\mathrm{G}$ in intestine [6]. Pure G and Glycyrrhetic acid (GA) have much higher risk of hypertension than the total extract of licorice [7]. This is due to the metabolic interaction of phenolic compounds and triterpenoids in the extract. Determination of the pharmacokinetic and metabolic interaction among these compounds is very important for the guidance of licorice application. Simultaneous quantification of phenolic compounds and triterpenoids is therefore strongly needed for researches on pharmaceutical mechanism of licorice and quality control of its preparations. Several attempts have been made to satisfy this requirement. These great jobs have been well summarized by Zhang and Ye [28]. HPLC is usually the most popular tool for natural products analysis. Three flavonoids and four triterpenoids in licorice have been determined by using a method of HPLC with UV detection, but had very long run time (more than $140 \mathrm{~min}$ for one run) [29]. Mass spectrometer is always combined with HPLC for complex sample analysis. This kind of approach is much more sensitive and selective. 42 compounds have been successfully identified by a method of HPLC UV TOF MS, but only three phenolic compounds and one triterpenoid among them were identified unambiguously [30]. Eight flavonoids have been successfully determined quantitatively by another HPLC MS/MS method [31]. HPLC MS is a relatively expensive instrument. The response range of electrospray ionization ion source (ESI) and some mass analyzers are sometimes not so broadened for quantification of multiple compounds with long dynamic range in contents, whereas in order to understand the mechanism of interactions among the ingredients of licorice during the process of decoction, absorption, metabolism, or at the physiological process, alternations of a series of compounds with similar structure in these processes were needed. More efficient methods that can quantify more compounds simultaneously are needed to satisfy this requirement.

Herein, a simple HPLC method, based on the different UV characters of triterpenoids, flavones, flavans, and chalcones, for simultaneous determination of glycyrrhizin and 15 flavonoids was developed and validated. Their contents in two wild licorices, prepared licorice, Sini Tang decoction, and the rat plasma after oral administration of Sini Tang decoction were estimated as a proof application.

\section{Material and Methods}

2.1. Materials and Sample Preparation. Two wild licorice samples were collected from different environments in northeast of Inner Mongolia by Professor Lijun Han, from Jilin Agricultural University, Changchun, Jilin Province. Both of them were identified to be the root of Glycyrrhiza uralensis Fisch. The wild licorice samples were dried under ambient temperature. Approximately $2 \mathrm{~g}$ powder of licorice was immersed in $20 \mathrm{~mL}$ of methanol at ambient temperature overnight before 30 minutes of treatment by an ultrasonic. The suspensions were filtered through $0.45 \mu \mathrm{m}$ pore size membrane and then. $20 \mu \mathrm{L}$ of filtration was finally applied to the HPLC analysis.

Honey-roasted licorice was bought from Beijing Tong Ren Tang Chinese Medicine Co., Ltd. It was identified to be the root of $G$. uralensis Fisch. The slice of the licorice was smashed to small pieces. The smashed honey-roasted-licorice was then processed in the same procedure with wild licorice.

Sini Tang decoction was composed and prepared according to the method described by Chinese Pharmacopoeia (2010 edition) [32]. It consisted of honey-roasted-licorice, Radix Aconiti Carmichaeli, and Rhizoma Zingiberis with a ratio of $3: 3: 2$. Both aconite and ginger were bought from China National Group Crop of Traditional and Herbal Medicine. Licorice is the same source as the previous. The decoction was made by three steps. The first step is the decoction of aconite and licorice together. $300 \mathrm{~g}$ aconite and $300 \mathrm{~g}$ licorice were mixed and immersed into $4000 \mathrm{~mL}$ water for 30 minutes. Approximately $1500 \mathrm{~mL}$ supernatant was taken out from the mixture after 120 minutes of boiling. The same volume of water was added into the residue then. About $1600 \mathrm{~mL}$ supernatant was taken out after the mixture was boiled for another 90 minutes. The second step is the decoction of zinger. $200 \mathrm{~g}$ dehydrated zinger was immersed into $1200 \mathrm{~mL}$ water for about 1 hour in a $2500 \mathrm{~mL}$ flask. The mixture was refluxed for 120 minutes to collect the volatile oil. Approximately $500 \mathrm{~mL}$ supernatant was taken out from the flask at the same time. $500 \mathrm{~mL}$ water was added into the zinger residue after that. All supernatants were taken out after another 90 minutes of boiling. The third step is Sini Tang decoction composing. All supernatants were mixed together, except the volatile oil which was added in to the mixture after it has been further treated. The mixture was concentrated to a volume of about $400 \mathrm{~mL}$ before $1200 \mathrm{~mL}$ alcohol $(95 \%$ ethanol) was mixed into the slurry. The precipitate was removed by filtration after about 24 hours. Alcohol was removed by distillation under reduced pressure then. The volatile oil was finally mixed into the extract, and then its volume was adjusted to $800 \mathrm{~mL}$ by water to make the final decoction. $200 \mu \mathrm{L}$ decoction was diluted to $5 \mathrm{~mL}$ for analysis. The diluted solution was filtered through a $0.45 \mu \mathrm{m}$ pore size membrane. $20 \mu \mathrm{L}$ of filtration was applied to HPLC analysis.

Plasma sample was kindly offered by Dr. Honhxia Liu. The plasma was collected from male Wistar rats, about $350 \mathrm{~g}$ weight. The rats had been subjected to an overnight fast. The blood was collected after 6 hours [33] of oral administration of Sini Tang decoction $(10 \mathrm{~mL} / \mathrm{kg}$ body weight, equal to $10 \mathrm{~g}$ herb per kg body weight). $1000 \mu \mathrm{L}$ of plasma was diluted with $3000 \mu \mathrm{L}$ of methanol. The precipitate of proteins in the plasma was removed by a centrifuge at a speed of 10000 rounds per minute (rpm). The supernatant was dried under a flow of nitrogen at ambient temperature. The residue was dissolved in $120 \mu \mathrm{L}$ methanol. $20 \mu \mathrm{L}$ of solution was applied to HPLC analysis.

2.2. Reagents and Standards. Compounds used as references shown in Table 1 were isolated and purified by Dr. Hongxia Liu $[34,35]$. The purities of them are all not less then 
TABLE 1: Glycyrrhizin and 15 flavonoids, their electrospray ionization mass and ultraviolet spectra data.

\begin{tabular}{|c|c|c|c|c|c|}
\hline Comp no. & Name & $M_{w}$ & $R_{t} / \mathrm{min}$ & MS/MS data & UV spectrum \\
\hline 1 & Liquiritigenin; 4'-O-glucopyranyl-(1-2)-glucopyranoside & 580 & 10.4 & $489,417,255$ & $\mathrm{~F}$ \\
\hline 2 & Sophoraflavone B & 416 & 14.2 & 415,253 & $\mathrm{C}$ \\
\hline 3 & Liquiritin apioside & 550 & 15.5 & $549,417,295,255$ & $\mathrm{~F}$ \\
\hline 4 & Liquiritigenin; 7,4'-O-diglucopyranoside & 580 & 15.8 & 417,255 & $\mathrm{~F}$ \\
\hline 5 & Liquiritin & 418 & 16.2 & $255,135,119$ & $\mathrm{~F}$ \\
\hline 6 & $6^{\prime \prime}$-O-a-hydroxypropynoylliquiritin & 490 & 18.4 & $417,399,327,255,135$ & $\mathrm{~F}$ \\
\hline 7 & IsoLiquiritin apioside & 550 & 19.6 & $549,429,417,357,327,297,255$ & $\mathrm{D}$ \\
\hline 8 & Isoliquiritin & 418 & 20.7 & $297,255,135,119$ & $\mathrm{D}$ \\
\hline 9 & Kumatakenin B & 254 & 21.8 & 253 & $\mathrm{C}$ \\
\hline 10 & Medicarpin-3-O-glucoside & 432 & 23.5 & $477,431,311,269$ & G \\
\hline 11 & Liquiritigenin & 256 & 24.3 & $255,153,135,119$ & $\mathrm{~F}$ \\
\hline 12 & Isoliquiritigenin & 256 & 30.9 & $255,153,135,119$ & $\mathrm{D}$ \\
\hline 13 & Formononetin & 268 & 31.7 & 267,252 & B \\
\hline 14 & Glycyrrhizin & 822 & 32.4 & $803,777,645,627,351$ & A \\
\hline 15 & Neoglycyrol & 366 & 41.0 & $365,350,307,295$ & $\mathrm{E}$ \\
\hline 16 & Isoglycyrol & 366 & 45.0 & 365,350 & $\mathrm{E}$ \\
\hline
\end{tabular}

90\%. Acetonitrile and methanol (both HPLC grade) were produced by Fisher, USA. Analytical grade formic acid was purchased from Beijing Chemical Factory.

2.3. Chromatography and ESI MS Analysis. HPLC UV ESI MS/MS was carried on an Agilent 1100 series LC MSD Trap, equipped with a diode array detector, a binary pump, a degasser, an Rheodyne valve manual sampler, and an ESI Trap MS. A gradient mobile phase was applied on a Phenomenex Luna C18 column $(4.6 \times 250 \mathrm{~mm}, 5 \mu \mathrm{m})$ for separation. The mobile phase consisted of water (with $0.1 \%$ formic acid, A) and acetonitrile (with $0.1 \%$ formic acid, B). The gradient was $0 \% \mathrm{~B}$ at 0 to 3 minutes the ratio of $\mathrm{B}$ increased linearly to $100 \%$ in 57 minutes then. The flow rate was $0.8 \mathrm{~mL} / \mathrm{min}$. HPLC chromatograms were recorded at $205 \mathrm{~nm}, 254 \mathrm{~nm}$, $280 \mathrm{~nm}, 310 \mathrm{~nm}$, and $355 \mathrm{~nm}$ with a gate width of $16 \mathrm{~nm}$ simultaneously in each run. UV spectra were obtained from 190 to $400 \mathrm{~nm}$.

ESI MS/MS analysis was acquired in negative mode. The fluent was split; about $200 \mu \mathrm{L}$ of eluent was introduced into the mass spectrometer per minute. The pressure of nebulizer gas was 0.21 mega-Pascal (MPa). The flow rate of dry gas was $8 \mathrm{~L} / \mathrm{min}$, and its temperature was $325^{\circ} \mathrm{C}$. The scan range of MS was from 100 to 1200 atom mass unit (amu). The target ion accumulated in the trap was 30000 counts. The maximum accumulation time was 50 milliseconds. The parameter of the trap was set in a smart mode. The target ion was $\mathrm{m} / z$ 350; the compound stability was $100 \%$; the trap drive level was $80 \%$. An auto MS2 mode was applied to obtain MS/MS spectra of different compounds. The MS/MS parameter was set as follows: mass range of parent ion was from 100 to $1200 \mathrm{amu} 1$ precursor was selected each cycle; each precursor would be excluded after 3 spectra; the release time was 0.5 minutes; the fragmentation voltage was optimized automatically from 0.3 to 2.0 electrical volt; the fragmentation time was 20

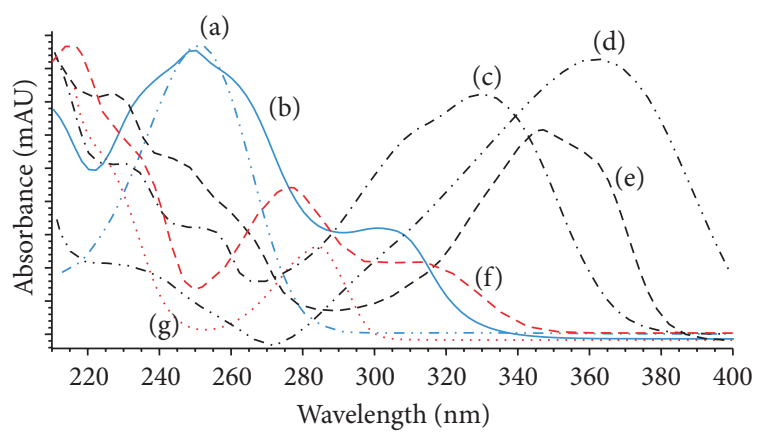

FIGURE 1: Ultraviolet spectra for different types of compounds. (a) Glycyrrhizin; (b) Formononetin; (c) Sophoraflavone B and Kumatakenin B; (d) Isoliquiritin apioside, Isoliquiritin, and Isoliquiritigenin; (e) Neoglycyrol and Isoglycyrol; (f) 4'-O-glucopyranyl-(1-2)-Liquiritigenin-glucopyranoside, Liquiritin apioside, 7,4'-OLiquiritigenin-diglucopyranoside, Liquiritin, 6"-O-a-hydroxypropynoylliquiritin, and Liquiritigenin; (g) Medicarpin-3-O-glucoside.

milliseconds; the activation width was $10 \mathrm{amu}$; the cutoff for daughter ion range was $27 \%$.

\section{Results and Discussion}

3.1. UV Spectrum Characters of the Target Analytes. All target analytes are shown in Table 1. These compounds can be divided into three groups according to their characters of UV spectroscopy. Their representative of UV spectra is also shown in Table 1 . The representative ultraviolet spectrum of each type is shown in Figure 1. The first group consists of Formononetin (13) and Glycyrrhizin (14) (spectra A and B). Their absorption peaks are both at approximately $254 \mathrm{~nm}$. The following seven compounds can be included in the second group: Sophoraflavone B (2) and Kumatakenin B (9), 


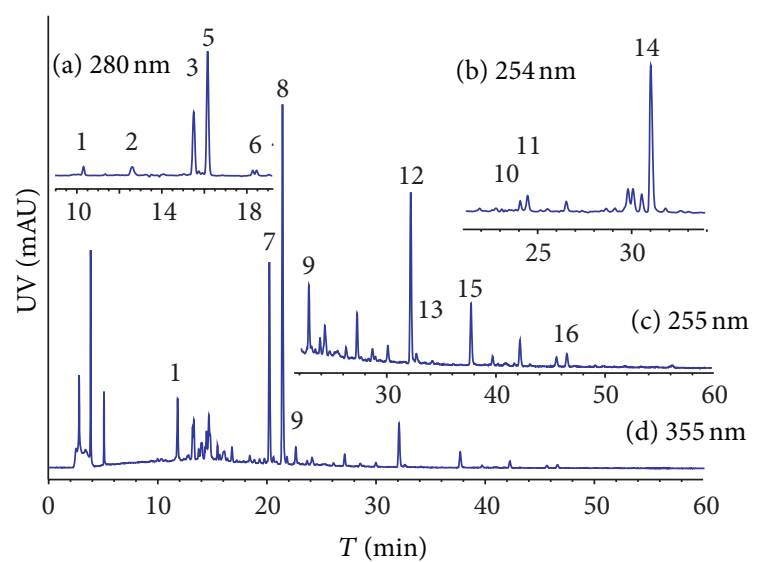

FIGURE 2: Representative chromatograms for licorice. Compounds $1,2,3,4,5$, and 6 were shown in a chromatogram recorded at $280 \mathrm{~nm}$; compounds $7,8,9,12,13,14$, and 16 were shown in a chromatogram recorded at $355 \mathrm{~nm}$; compounds 10,11 , and 14 were shown in a chromatogram recorded at $254 \mathrm{~nm}$; all chromatograms shown were recorded in the same run.

Isoliquiritin apioside (7), Isoliquiritin (8), Isoliquiritigenin (12), Neoglycyrol (15), and Isoglycyrol (16) (2 and 9 spectra $C ; 7,8$, and 12 spectra $D ; 15$ and 16 spectra E). The maximum UV absorption of these compounds ranged from $330 \mathrm{~nm}$ to $370 \mathrm{~nm}$, at a much longer wavelength, where the compounds from the other two groups have no absorbance. Additionally, all compounds in the second group have an absorption valley at about $280 \mathrm{~nm}$. The third group includes mainly liquiritigenin and its derivatives (1,3 6, and 11), and a flavan (Medicarpin-3-O-glucoside 10). These compounds have maximum a absorption at about $280 \mathrm{~nm}$, the absorption valley of compounds in group two as well as an absorption valley at approximately $254 \mathrm{~nm}$, the maximum absorption of compounds in group one. In view of this, compounds from different groups can be distinguished from each other by detecting at different wavelengths, 254, 280, and $355 \mathrm{~nm}$, when they are coeluted.

3.2. Separation and Selectivity. Representative chromatogram of all 16 target analytes in licorice is shown in Figure 2. All 16 compounds can be the baseline separated from the background constituents in licorice in a related short run time $(60 \mathrm{~min})$ when the detector response was recorded at a certain wavelength for each type of compound. The selectivity of the method was further proved by HPLC ESI MS analysis and purity analyses of HPLC UV peaks. The MS/MS data of 16 target analytes is shown in Table 1. Peak purities of all target analytes were larger than 950 at the range of their detection wavelengths. Peak purities of compounds $2,3,5,6,7,8,14,15$, and 16 were also proved by the MS spectra obtained at the range of the peaks. Compound 1 was coeluted with a flavone-C-glycoside (according to its MS/MS data $[36,37])$. The ultraviolet spectrum of a flavone is $\mathrm{C}$ type (Figure 1), which may interfere with the detection of compound 1 (spectrum F) theoretically. However, the purity analysis of chromatographic peak of compound 1 indicated

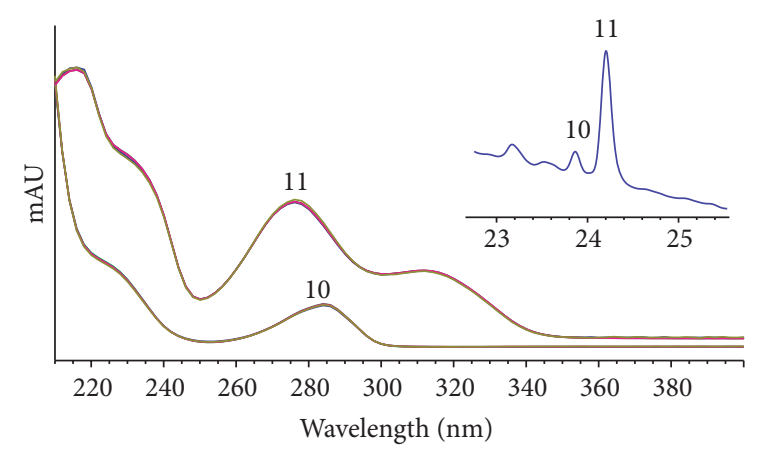

FIGURE 3: The representative chromatograms and their UV spectra for Medicarpin-3-O-glucoside (10) and Liquiritigenin (11). (a) Chromatogram recorded at $205 \mathrm{~nm}$. (b) Overlay of UV spectra at 5 data points on each chromatographic peak at $205 \mathrm{~nm}$ for compounds 10 and 11.

that all spectra in the range of the peak were well overlapped. The purity of the chromatographic peak of compound 1 was tested at wave width that ranged from 200 to $400 \mathrm{~nm}$ with a purity factor of more than 950 . The coeluted flavone-Cglycoside did not affect the detection. This may be because the concentration of the coeluted flavone was very low. compound 1 can be precisely determined in this distance. compounds 3 and 5 may interfere with the detection of compound 4 a little. The UV spectrum of compound 4 is the same as them; so it is not able to be distinguished from compounds 3 and 5 by detecting at different wavelengths. But they exhibited near the baseline separation. The resolution factors of compounds 4 to 3 and 5 are 1.41 1.52 and 2.43 2.44 , respectively. The chromatographic peaks of compounds 12 and 13 were partly overlapped with that of compound 14 (glycyrrhizin). Compounds 12 and 13 can be precisely quantitatively determined without interference from compound 14 by detecting at 355 and $310 \mathrm{~nm}$, where compound 14 has no absorbance. Concentration of compound 14 was much higher in the samples than the other two; the estimation of its content was not affected by coeluted compounds 12 and 13. Ions represent glycyrrhizin type compounds (the production of a daughter ion $m / z 351$, which represents the structure of the glycoside chain [38]) that were recognized in the MS spectra of compounds $9(\mathrm{~m} / z 823,837,969$, and 989), $10(\mathrm{~m} / z$ 809, 821 , and 983), $11(\mathrm{~m} / z 821$ and 983), but the detection of these compounds was not interfered by the coeluted constituents by detecting at $355 \mathrm{~nm}, 280 \mathrm{~nm}$, and $280 \mathrm{~nm}$, respectively. However, Medicarpin-3-O-glucoside (10) was estimated with its detector response at $205 \mathrm{~nm}$ because its detector response at $280 \mathrm{~nm}$ is too weak to be recognized in real samples for its low concentration. The representative chromatogram for 10 as well as its peak purity analysis is shown in Figure 3.

3.3. Linearity, Recovery, and Precision. The detector response against compound mass perinjection was used to establish the linearity of the method. Due to the large dynamic content range of those compounds in licorice, the linearity covered ranges for different compounds were quite a little different from each other. The linearity experiment for each analyte 
TABLE 2: Linearity and recovery of all target analytes.

\begin{tabular}{|c|c|c|c|c|c|c|}
\hline \multirow{2}{*}{ No. } & \multicolumn{4}{|c|}{ Linearity $(n=3)$} & \multirow{2}{*}{ Recovery $(\%, n=2)$} & \multirow{2}{*}{ Wave length (nm) } \\
\hline & Slop & Intercept & $R^{2}$ & Range ( $\mu \mathrm{g} / \mathrm{inj})$ & & \\
\hline 1 & $39.9310 \pm 0.1047$ & $-5.9005 \pm 1.4168$ & $0.9964 \pm 0.00107$ & $0.122 \sim 9.760$ & $90.7 \pm 1.4$ & 280 \\
\hline 2 & $100.0500 \pm 0.1735$ & $-16.1570 \pm 2.5922$ & $0.9991 \pm 0.00045$ & $0.135 \sim 10.800$ & $92.4 \pm 3.4$ & 310 \\
\hline 3 & $312.7770 \pm 0.5550$ & $-108.1800 \pm 31.7830$ & $0.9998 \pm 0.00006$ & $0.550 \sim 55.000$ & $97.7 \pm 3.3$ & 280 \\
\hline 4 & $178.7300 \pm 0.3786$ & $-82.4700 \pm 7.6173$ & $0.9934 \pm 0.00038$ & $0.186 \sim 18.600$ & $88.7 \pm 3.8$ & 280 \\
\hline 5 & $513.2200 \pm 0.5100$ & $-140.5100 \pm 15.0200$ & $0.9995 \pm 0.00006$ & $0.282 \sim 28.200$ & $97.6 \pm 1.8$ & 280 \\
\hline 6 & $86.7590 \pm 0.1000$ & $-34.8500 \pm 1.5015$ & $0.9896 \pm 0.00010$ & $0.166 \sim 16.600$ & $93.3 \pm 1.0$ & 280 \\
\hline 7 & $121.7400 \pm 0.0404$ & $-26.2100 \pm 1.0551$ & $0.9985 \pm 0.00010$ & $0.240 \sim 19.200$ & $91.8 \pm 0.2$ & 355 \\
\hline 8 & $511.4600 \pm 0.3550$ & $41.6910 \pm 11.6620$ & $0.9964 \pm 0.00000$ & $0.295 \sim 23.600$ & $100.3 \pm 1.4$ & 355 \\
\hline 9 & $75.3170 \pm 0.1334$ & $-10.4140 \pm 4.7618$ & $0.9989 \pm 0.00030$ & $0.338 \sim 27.040$ & $96.8 \pm 1.9$ & 355 \\
\hline 10 & $64.3160 \pm 0.0153$ & $-6.5371 \pm 1.7859$ & $0.9990 \pm 0.00021$ & $0.263 \sim 21.040$ & $94.9 \pm 0.4$ & 205 \\
\hline 11 & $164.7600 \pm 0.1429$ & $-18.8230 \pm 4.1025$ & $0.9988 \pm 0.00015$ & $0.265 \sim 21.200$ & $94.2 \pm 0.2$ & 280 \\
\hline 12 & $405.4600 \pm 0.0900$ & $-88.4210 \pm 8.1045$ & $0.9993 \pm 0.00031$ & $0.260 \sim 13.000$ & $96.7 \pm 1.6$ & 355 \\
\hline 13 & $73.9750 \pm 0.0976$ & $-8.1110 \pm 2.3016$ & $0.9988 \pm 0.00023$ & $0.251 \sim 20.080$ & $94.8 \pm 0.7$ & 310 \\
\hline 14 & $840.7600 \pm 0.1600$ & $-45.1990 \pm 2.0570$ & $0.9977 \pm 0.00000$ & $0.123 \sim 12.300$ & $105.3 \pm 0.4$ & 254 \\
\hline 15 & $100.5700 \pm 0.1375$ & $-10.9290 \pm 2.8705$ & $0.9988 \pm 0.00025$ & $0.194 \sim 15.520$ & $95.4 \pm 1.4$ & 355 \\
\hline 16 & $93.4020 \pm 5.8669$ & $-2.5702 \pm 0.8098$ & $0.9988 \pm 0.00020$ & $0.071 \sim 5.680$ & $94.7 \pm 1.1$ & 355 \\
\hline
\end{tabular}

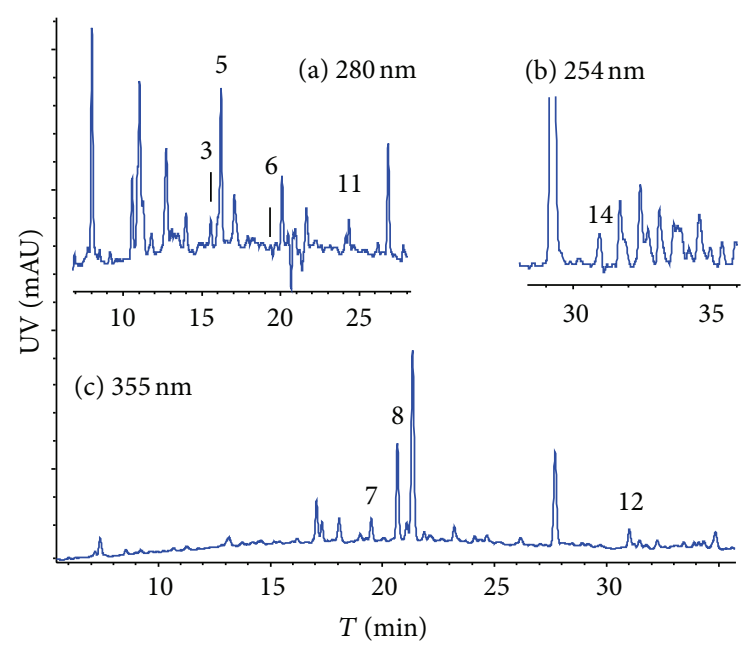

FIgURE 4: Representative chromatograms for plasma from rat administered Sini Tang decoction. Compounds 3, 5, 6, and 11 were shown in a chromatogram recorded at $280 \mathrm{~nm}$; compounds 7,8 , and 12 were shown in a chromatogram recorded at $355 \mathrm{~nm}$; compound 14 was shown in a chromatogram recorded at $254 \mathrm{~nm}$; all chromatograms shown were recorded in the same run.

was triplicate. The fit of linearity for each compound was good. The linearity correlation coefficients $\left(R^{2}\right)$ of all 16 target analytes varied from 0.9896 to 0.9998 , with standard derivations ranging from $0.000 \%$ to $0.107 \%$, respectively (shown in Table 2). The slope of each compound was stable (RSD, $0.019 \% \sim 6.281 \%, n=3$ ).

The recovery of the methanol extracting method was estimated by adding known amounts of compounds into the powder of licorice. The difference between spiked licorice and nonspiked licorice was used to define the recovery of the method. The recoveries of all 16 target analytes are also shown in Table 2. The average recoveries were quite close to $100 \%$ for each compound at the detected amount.

The relative standard derivation (RSD) of the three analyses of the mixture of 16 compounds was used for determining the precision of the method. According to the results shown in Table 2, the precision of the method was good. None of them was more than $6.3 \%$ when the injected amounts for compounds 1 to 16 were $0.122,0.270,2.750,0.372,1.410,0.166$, $0.480,1.475,0.135,0.263,0.265,1.300,0.100,2.460,0.097$, and $0.028 \mu \mathrm{g}$ per injection, respectively.

3.4. Determination of 16 Target Analytes in Licorices and Sini Tang Decoction. Figure 3 shows the representative chromatograms of different licorices. The separation and selectivity of the detection are stated in Section 3.2. The concentrations of these compounds were determined by comparing with the external references. The contents of all 16 compounds in two wild licorices, prepared licorice, and Sini Tang decoction are shown in Table 3. The highest content is glycyrrhizin in one of the wild licorices. The minimum content detected is Formononetin in Sini Tang decoction. The amount of glycyrrhizin in wild licorice was consistent with the results in the literature $[29,39]$.

3.5. Determination of Constituents from Licorice in Rat Plasma. The constituent from licorice in rat plasma, which was orally administered Sini Tang decoction, was also estimated in this work. The experiment was carried out only one time. Seven constituents, including Liquiritin apioside (3), Liquiritin (5), Isoliquiritin apioside (7), Isoliquiritin (8), Liquiritigenin (11), Isoliquiritigenin (12), and, Glycyrrhizin (14), were unambiguously detected (Figure 4). Their concentrations were estimated by comparing to the external references (shown in Table 3). Nine of 16 target analytes had not been detected in the plasma. This may be because the dose 
TABLE 3: Contents of all 16 compounds in different sample $(n=3)$.

\begin{tabular}{|c|c|c|c|c|c|c|c|c|}
\hline No. & $\begin{array}{l}\text { licorice } 1 \\
(\mathrm{mg} / \mathrm{g})\end{array}$ & $\begin{array}{c}\text { licorice } 2 \\
(\mathrm{mg} / \mathrm{g})\end{array}$ & $\begin{array}{l}\text { R-licorice } \\
(\mathrm{mg} / \mathrm{g})\end{array}$ & $\begin{array}{l}\text { gly-D } \\
(\mathrm{mg} / \mathrm{g}) \\
\end{array}$ & $\operatorname{sini}-\mathrm{D}^{*}(\mathrm{mg} / \mathrm{g})$ & $\begin{array}{l}\text { Serum } \\
(\mathrm{mg} / \mathrm{L})\end{array}$ & $\begin{array}{c}\text { gly-D/gly-E } \\
(\%)\end{array}$ & $\begin{array}{c}\text { sini/gly-E } \\
(\%)\end{array}$ \\
\hline 1 & $3.296 \pm 0.302$ & $1.536 \pm 0.100$ & $0.526 \pm 0.034$ & $0.352 \pm 0.032$ & $0.576 \pm 0.037$ & nd & $66.9 \pm 5.7$ & $109.5 \pm 7.5$ \\
\hline 2 & $0.238 \pm 0.251$ & $0.166 \pm 0.200$ & $0.580 \pm 0.076$ & $0.113 \pm 0.004$ & $0.077 \pm 0.010$ & nd & $19.5 \pm 0.7$ & $13.2 \pm 1.7$ \\
\hline 3 & $12.382 \pm 1.110$ & $13.892 \pm 0.489$ & $8.587 \pm 0.302$ & $1.796 \pm 0.161$ & $3.843 \pm 0.135$ & 1.190 & $20.9 \pm 1.9$ & $44.8 \pm 1.6$ \\
\hline 4 & $0.299 \pm 0.036$ & $0.129 \pm 0.012$ & $0.220 \pm 0.020$ & $0.071 \pm 0.009$ & $0.224 \pm 0.020$ & nd & $32.2 \pm 4.5$ & $101.9 \pm 9.1$ \\
\hline 5 & $10.488 \pm 0.696$ & $8.631 \pm 0.316$ & $9.838 \pm 0.360$ & $1.956 \pm 0.130$ & $4.352 \pm 0.159$ & 1.387 & $19.9 \pm 1.3$ & $44.2 \pm 1.6$ \\
\hline 6 & nd & nd & $0.143 \pm 0.005$ & $0.207 \pm 0.014$ & $0.082 \pm 0.003$ & 0.127 & $144.6 \pm 7.1$ & $57.3 \pm 2.1$ \\
\hline 7 & $6.757 \pm 0.342$ & $4.943 \pm 0.133$ & $3.608 \pm 0.097$ & $0.567 \pm 0.029$ & $0.962 \pm 0.026$ & 0.297 & $15.7 \pm 0.8$ & $26.7 \pm 0.8$ \\
\hline 8 & $1.515 \pm 0.103$ & $1.051 \pm 0.040$ & $2.471 \pm 0.095$ & $0.526 \pm 0.036$ & $0.959 \pm 0.037$ & 0.071 & $21.3 \pm 1.6$ & $38.8 \pm 1.6$ \\
\hline 9 & $0.269 \pm 0.006$ & $0.093 \pm 0.060$ & $0.045 \pm 0.029$ & $0.010 \pm 0.000$ & $0.034 \pm 0.022$ & nd & $22.8 \pm 19.8$ & $75.2 \pm 40.0$ \\
\hline 10 & nd & $2.059 \pm 0.023$ & $0.026 \pm 0.000$ & $0.006 \pm 0.000$ & $0.041 \pm 0.000$ & nd & $25.3 \pm 5.0$ & $160.7 \pm 5.0$ \\
\hline 11 & $1.437 \pm 0.083$ & $0.292 \pm 0.040$ & $1.631 \pm 0.222$ & $0.158 \pm 0.009$ & $0.384 \pm 0.052$ & 0.103 & $9.7 \pm 0.6$ & $23.5 \pm 3.1$ \\
\hline 12 & $0.833 \pm 0.016$ & nd & $0.371 \pm 0.012$ & $0.125 \pm 0.002$ & $0.364 \pm 0.007$ & 0.357 & $33.7 \pm 2.7$ & $98.2 \pm 2.7$ \\
\hline 13 & $0.162 \pm 0.019$ & $0.033 \pm 0.001$ & $0.107 \pm 0.003$ & $0.008 \pm 0.001$ & $0.023 \pm 0.001$ & nd & $7.4 \pm 5.0$ & $21.6 \pm 5.0$ \\
\hline 14 & $34.451 \pm 2.314$ & $26.719 \pm 0.464$ & $28.839 \pm 0.501$ & $5.357 \pm 0.360$ & $11.441 \pm 0.199$ & 2.463 & $18.6 \pm 1.2$ & $39.7 \pm 0.7$ \\
\hline 15 & $0.471 \pm 0.032$ & $0.323 \pm 0.056$ & $0.120 \pm 0.021$ & $0.012 \pm 0.001$ & $0.022 \pm 0.004$ & nd & $10.3 \pm 1.4$ & $18.7 \pm 5.0$ \\
\hline 16 & nd & nd & $0.087 \pm 0.015$ & $0.006 \pm 0.000$ & $0.004 \pm 0.000$ & nd & $7.2 \pm 2.6$ & $5.1 \pm 3.0$ \\
\hline
\end{tabular}

${ }^{*}$ Contents were estimated based on the mass of licorice in the mixture; R-licorice, honey-roasted-licorice; Sini-D, decoction of Sini Tang.

administered should be a little low for plasma metabolites analysis requirements according to the previous work $[5,33]$. But this work provided a stable proof in which this method can be used for simultaneous determination of the above mentioned compounds in biosample.

\section{Conclusion}

A simple and reproducible HPLC UV method was reported for simultaneous determination of sixteen constituents in licorice and its products. This method was proved to be fit for biosample analysis. It will provide a tool for chemical and pharmacological studies of licorice.

\section{Conflict of Interests}

All herbal materials used in the current research were purchased from local markets with no conflict of interests related to the manufacturers.

\section{Authors' Contribution}

Yongqian Zhang and Jin Cao had equal contribution in this paper.

\section{Acknowledgments}

This work was supported by the Beijing Institute of Technology Basic Research Foundation no. 20091642014, and Jilin Province Science and Technology Development Foundation (20096040).

\section{References}

[1] V. Nandakumar, T. Singh, and S. K. Katiyar, "Multi-targeted prevention and therapy of cancer by proanthocyanidins," Cancer Letters, vol. 269, no. 2, pp. 378-387, 2008.

[2] A. Goel, S. Jhurani, and B. B. Aggarwal, "Multi-targeted therapy by curcumin: how spicy is it?" Molecular Nutrition and Food Research, vol. 52, no. 9, pp. 1010-1030, 2008.

[3] H. Wagner and G. Ulrich-Merzenich, "Synergy research: approaching a new generation of phytopharmaceuticals," Phytomedicine, vol. 16, no. 2-3, pp. 97-110, 2009.

[4] H. Wagner, "Synergy research: approaching a new generation of phytopharmaceuticals," Fitoterapia, vol. 82, no. 1, pp. 34-37, 2011.

[5] C. Xiang, X. Qiao, Q. Wang et al., "From single compounds to herbal extract: a strategy to systematically characterize the metabolites of licorice in rats," Drug Metabolism and Disposition, vol. 39, no. 9, pp. 1597-1608, 2011.

[6] Y.-C. Hou, S.-L. Hsiu, H. Ching et al., "Profound difference of metabolic pharmacokinetics between pure glycyrrhizin and glycyrrhizin in licorice decoction," Life Sciences, vol. 76, no. 10, pp. 1167-1176, 2005.

[7] G. Cantelli-Forti, M. A. Raggi, F. Bugamelli, F. Maffei, A. Villari, and N. M. Trieff, "Toxicological assessment of liquorice: biliary excretion in rats," Pharmacological Research, vol. 35, no. 5, pp. 463-470, 1997.

[8] M. A. Raggi, F. Maffei, F. Bugamelli, and G. Cantelli Forti, "Bioavailability of glycyrrhizin and licorice extract in rat and human plasma as detected by a HPLC method," Die Pharmazie, vol. 49, no. 4, pp. 269-272, 1994.

[9] S.-P. Lin, S.-Y. Tsai, Y.-C. Hou, and P.-D. L. Chao, "Glycyrrhizin and licorice significantly affect the pharmacokinetics of methotrexate in rats," Journal of Agricultural and Food Chemistry, vol. 57, no. 5, pp. 1854-1859, 2009. 
[10] S.-Y. Xiao, H.-X. Liu, W.-H. Lin, and J.-S. Yang, "Diarylheptanoids in rhizoma zingiberis and their stereoslective reactions during the process of decocting," Chinese Journal of Analytical Chemistry, vol. 35, no. 9, pp. 1295-1300, 2007.

[11] M. V. Palagina, N. S. Dubnyak, I. N. Dubnyak, and P. S. Zorikov, "Correction of respiratory organ impairment with ural licorice preparations in chronic skin diseases," Terapevticheskii Arkhiv, vol. 75, no. 1, pp. 63-65, 2003.

[12] B. Liu, J. Yang, Q. Wen, and Y. Li, "Isoliquiritigenin, a flavonoid from licorice, relaxes guinea-pig tracheal smooth muscle in vitro and in vivo: role of cGMP/PKG pathway," European Journal of Pharmacology, vol. 587, no. 1-3, pp. 257-266, 2008.

[13] B. Jayaprakasam, S. Doddaga, R. Wang, D. Holmes, J. Goldfarb, and X.-M. Li, "Licorice flavonoids inhibit eotaxin-1 secretion by human fetal lung fibroblasts in vitro," Journal of Agricultural and Food Chemistry, vol. 57, no. 3, pp. 820-825, 2009.

[14] Y.-C. Xie, X.-W. Dong, X.-M. Wu, X.-F. Yan, and Q.-M. Xie, "Inhibitory effects of flavonoids extracted from licorice on lipopolysaccharide-induced acute pulmonary inflammation in mice," International Immunopharmacology, vol. 9, no. 2, pp. 194200, 2009.

[15] A. Agarwal, D. Gupta, G. Yadav, P. Goyal, P. K. Singh, and U. Singh, "An evaluation of the efficacy of licorice gargle for attenuating postoperative sore throat: a prospective, randomized, single-blind study," Anesthesia and Analgesia, vol. 109, no. 1, pp. 77-81, 2009.

[16] M. Katayama, T. Fukuda, T. Okamura et al., "Effect of dietary addition of seaweed and licorice on the immune performance of pigs," Animal Science Journal, vol. 82, no. 2, pp. 274-281, 2011.

[17] H. Chang, B.-B. Su, Y.-P. Zhou, and D.-R. He, "Assortativity and act degree distribution of some collaboration networks," Physica A, vol. 383, no. 2, pp. 687-702, 2007.

[18] M. Schambelan, "Licorice ingestion and blood pressure regulating hormones," Steroids, vol. 59, no. 2, pp. 127-130, 1994.

[19] B. R. Walker and C. R. W. Edwards, "Licorice-induced hypertension and syndromes of apparent mineralocorticoid excess," Endocrinology and Metabolism Clinics of North America, vol. 23, no. 2, pp. 359-377, 1994.

[20] C. Schulze zur Wiesch, N. Sauer, and J. Aberle, "Hypertension and hypokalemia-a reninoma as the cause of suspected liquorice-induced arterial hypertension," Deutsche Medizinische Wochenschrift, vol. 136, no. 17, pp. 882-884, 2011.

[21] H. E. Miettinen, K. Piippo, T. Hannila-Handelberg et al., "Licorice-induced hypertension and common variants of genes regulating renal sodium reabsorption," Annals of Medicine, vol. 42, no. 6, pp. 465-474, 2010.

[22] P. Pant, L. Nadimpalli, M. Singh, and J. C. Cheng, "A case of severe hypokalemic paralysis and hypertension. Licoriceinduced hypokalemic paralysis," American Journal of Kidney Diseases, vol. 55, no. 6, pp. A35-A37, 2010.

[23] A. S. Cooney and J. T. Fitzsimons, "Increased sodium appetite and thirst in rat induced by the ingredients of liquorice, glycyrrhizic acid and glycyrrhetinic acid," Regulatory Peptides, vol. 66, no. 1-2, pp. 127-133, 1996.

[24] N. Mumoli and M. Cei, "Licorice-induced hypokalemia," International Journal of Cardiology, vol. 124, no. 3, pp. e42-e44, 2008.

[25] C. Noguchi, J. Yang, K. Sakamoto et al., "Inhibitory effects of isoliquiritigenin and licorice extract on voltage-dependent $\mathrm{K}^{+}$ currents in H9c2 cells," Journal of Pharmacological Sciences, vol. 108, no. 4, pp. 439-445, 2008.
[26] J. Hukkanen, O. Ukkola, and M. J. Savolainen, "Effects of lowdose liquorice alone or in combination with hydrochlorothiazide on the plasma potassium in healthy volunteers," Blood Pressure, vol. 18, no. 4, pp. 192-195, 2009.

[27] G. Chen, L. Zhu, Y. Liu, Q. Zhou, H. Chen, and J. Yang, "Isoliquiritigenin, a flavonoid from licorice, plays a dual role in regulating gastrointestinal motility in vitro and in vivo," Phytotherapy Research, vol. 23, no. 4, pp. 498-506, 2009.

[28] Q. Zhang and M. Ye, "Chemical analysis of the Chinese herbal medicine Gan-Cao (licorice)," Journal of Chromatography A, vol. 1216, no. 11, pp. 1954-1969, 2009.

[29] Y.-C. Wang and Y.-S. Yang, "Simultaneous quantification of flavonoids and triterpenoids in licorice using HPLC," Journal of Chromatography B, vol. 850, no. 1-2, pp. 392-399, 2007.

[30] G. Tan, Z. Zhu, H. Zhang et al., "Analysis of phenolic and triterpenoid compounds in licorice and rat plasma by highperformance liquid chromatography diode-array detection, time-of-flight mass spectrometry and quadrupole ion trap mass spectrometry," Rapid Communications in Mass Spectrometry, vol. 24, no. 2, pp. 209-218, 2010.

[31] L. Li, S. Liang, F. Du, and C. Li, "Simultaneous quantification of multiple licorice flavonoids in rat plasma," Journal of the American Society for Mass Spectrometry, vol. 18, no. 4, pp. 778782, 2007.

[32] "Sini Tang," in Chinese Pharmacopoeia, Chinese Pharmacopoeia Committee, Ed., p. 650, Chinese Press of Pharmaceutical and Medicinal Science and Technology, Beijing, China, 2010.

[33] Y. Ozaki, M. Noguchi, H. Kamakura, and M. Harada, "Studies on concentration of glycyrrhizin in plasma and its absorption after oral administration of licorice extract and glycyrrhizin," Yakugaku Zasshi, vol. 110, no. 1, pp. 77-81, 1990.

[34] H.-X. Liu, W.-H. Lin, X.-L. Wang, and J.-S. Yang, "Flavonoids from preparation of traditional Chinese medicines named SiniTang," Journal of Asian Natural Products Research, vol. 7, no. 2, pp. 139-143, 2005.

[35] H.-X. Liu, W.-H. Lin, and J.-S. Yang, "Studies on chemical constituents of sini tang," China Journal of Chinese Materia Medica, vol. 29, no. 5, pp. 434-436, 2004.

[36] A. P. Rauter, A. Martins, C. Borges et al., "Liquid chromatography-diode array detection-electrospray ionisation mass spectrometry/nuclear magnetic resonance analyses of the anti-hyperglycemic flavonoid extract of Genista tenera: structure elucidation of a flavonoid-C-glycoside," Journal of Chromatography A, vol. 1089, no. 1-2, pp. 59-64, 2005.

[37] P. Waridel, J.-L. Wolfender, K. Ndjoko, K. R. Hobby, H. J. Major, and K. Hostettmann, "Evaluation of quadrupole time-of-flight tandem mass spectrometry and ion-trap multiple-stage mass spectrometry for the differentiation of C-glycosidic flavonoid isomers," Journal of Chromatography A, vol. 926, no. 1, pp. 2941, 2001.

[38] M. Jin, Y. Yang, B. Su, and Q. Ren, "Determination of soyasaponins $\mathrm{Ba}$ and $\mathrm{Bb}$ in human serum by high-performance liquid chromatography coupled with electrospray ionization tandem mass spectrometry," Journal of Chromatography B, vol. 846, no. 1-2, pp. 169-175, 2007.

[39] S. Tsukamoto, M. Aburatani, T. Yoshida, Y. Yamashita, A. A. ElBeih, and T. Ohta, "CYP3A4 inhibitors isolated from licorice," Biological and Pharmaceutical Bulletin, vol. 28, no. 10, pp. 20002002, 2005 

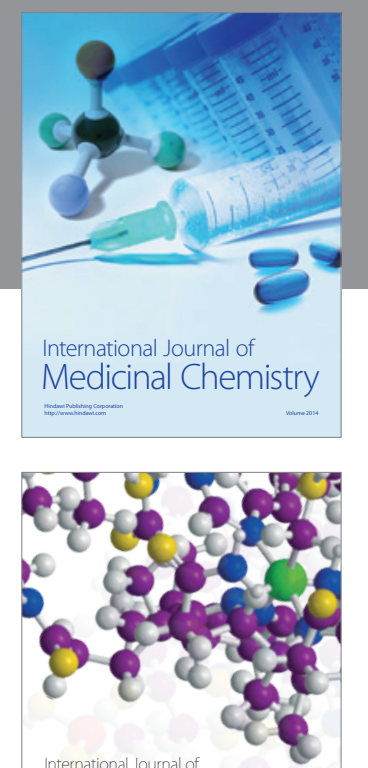

\section{Carbohydrate} Chemistry

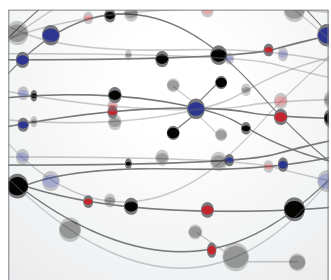

The Scientific World Journal
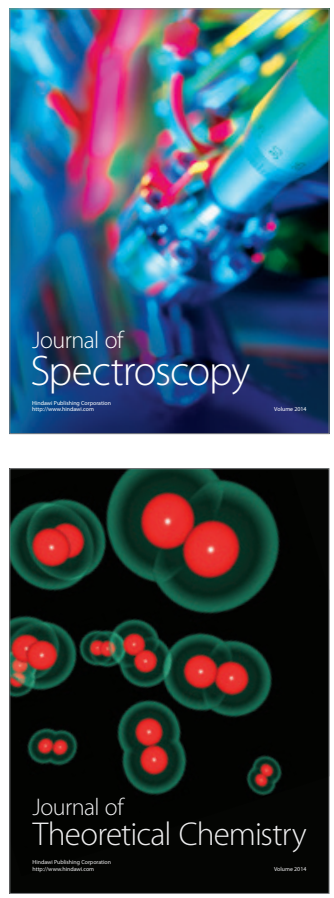
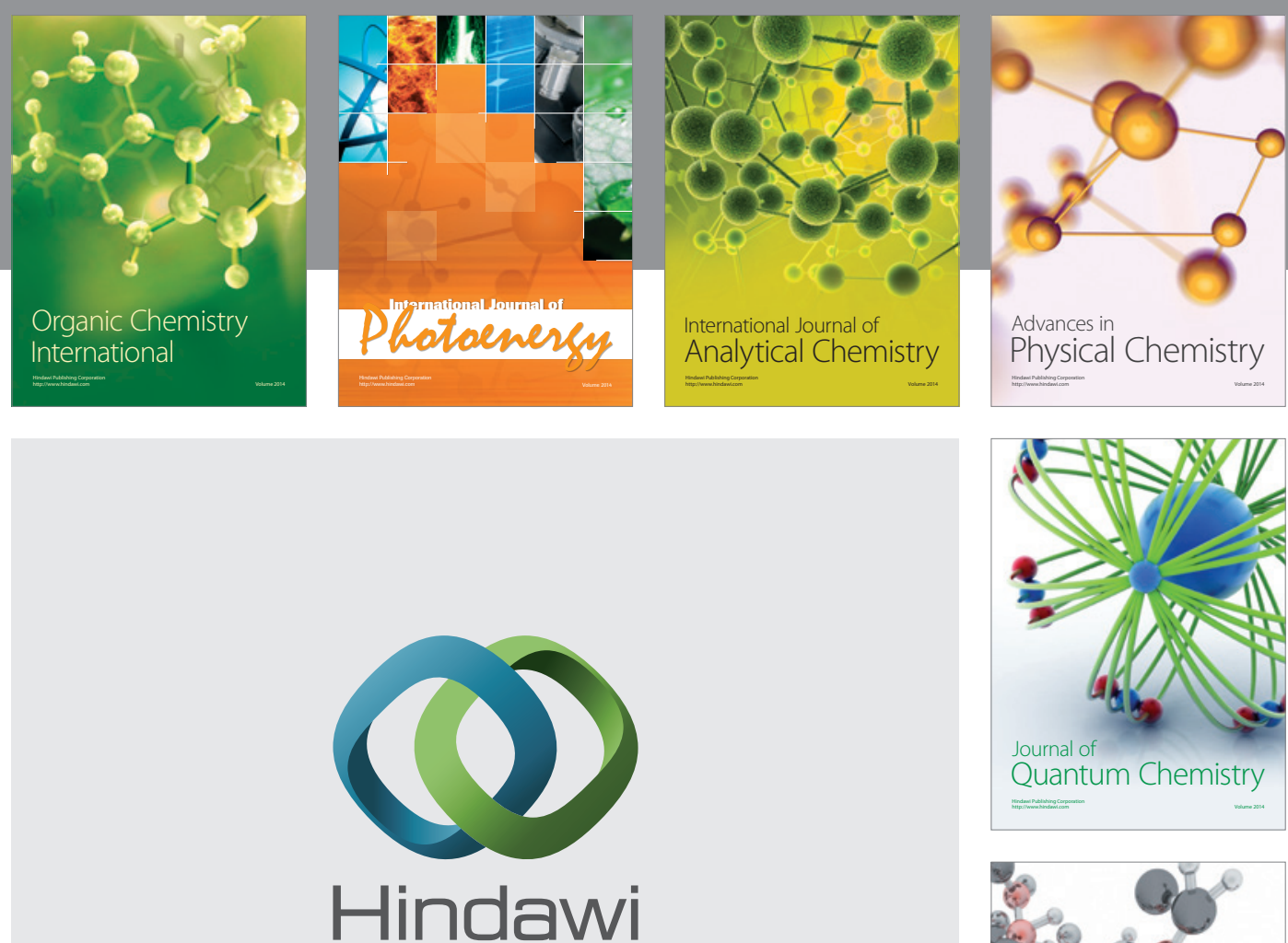

Submit your manuscripts at

http://www.hindawi.com

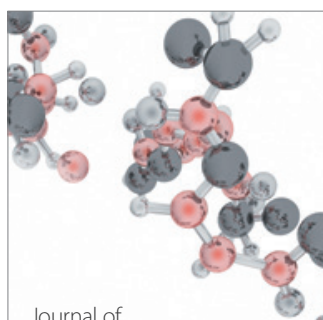

Analytical Methods

in Chemistry

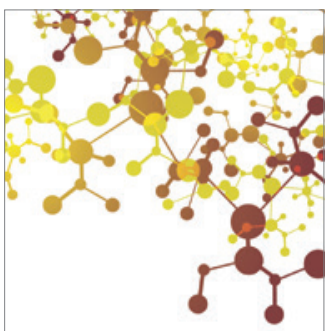

Journal of

Applied Chemistry

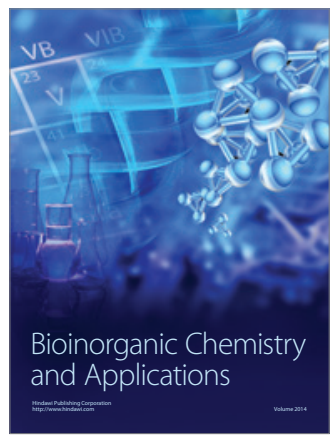

Inorganic Chemistry
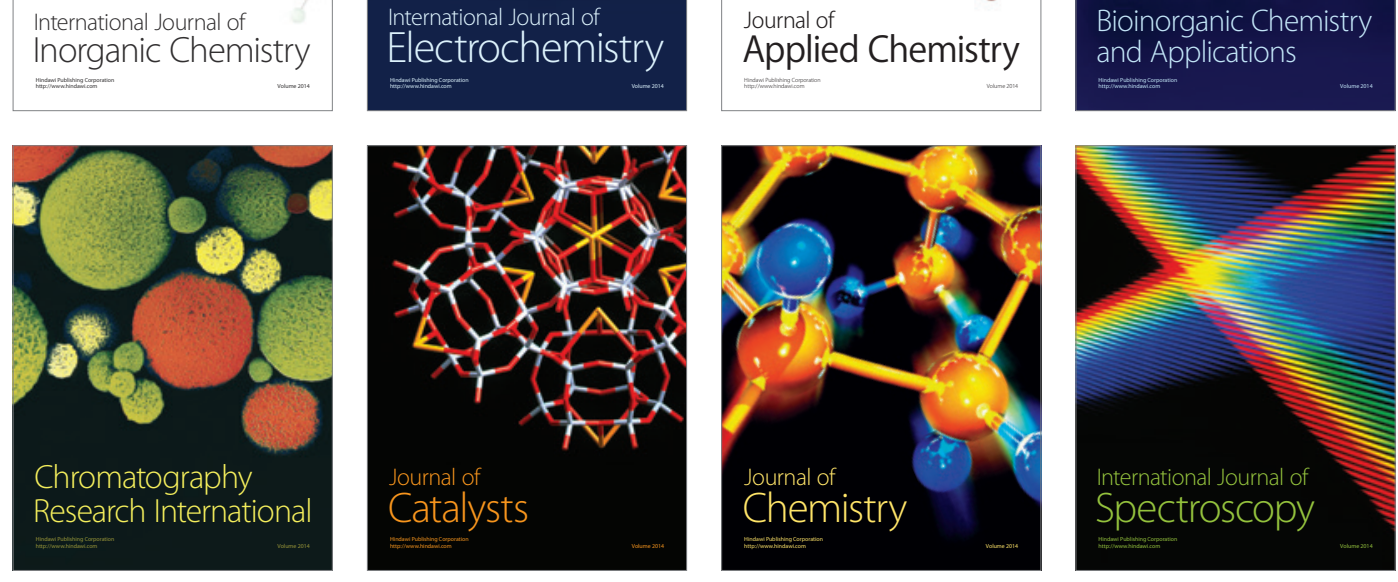